\title{
A Discrete Analysis of Non-reflecting Boundary Conditions for Discontinuous Galerkin Method
}

\author{
Fang Q. $\mathrm{Hu}^{*}$ \\ Department of Mathematics and Statistics \\ Old Dominion University, Norfolk, Virginia 23529 \\ Harold L. Atkins ${ }^{\dagger}$ \\ NASA Langley Research Center \\ Hampton, Virginia 23681
}

\begin{abstract}
We present a discrete analysis of non-reflecting boundary conditions for the discontinuous Galerkin method. The boundary conditions considered in this paper include the recently proposed Perfectly Matched Layer absorbing boundary condition for the linearized Euler equation and two non-reflecting boundary conditions based on the characteristic decomposition of the flux on the boundary. The analyses for the three boundary conditions are carried out in a unifled way. In each case, eigensolutions of the discrete system are obtained and applied to compute the numerical reflection coefficients of a specified out-going wave. The dependencies of the reflections at the boundary on the out-going wave angle and frequency as well as the mesh sizes are studied. Comparisons with direct numerical simulation results are also presented.
\end{abstract}

\section{Introduction}

The discontinuous Galerkin method (DGM) provides an attractive platform for computational aeroacoustics in dealing with complex geometries while using high order approximations [3]. Our recent works have shown that discontinuous Galerkin scheme is super-accurate for numerical simulation of wave propagations [6,7]. A framework of discrete analysis has been proposed in $[6,7]$ to study systematically and analytically the numerical errors occurred due to a sudden discontinuity in mesh topology or order of the basis functions in one and two spatial dimensions. In this paper, this framework is extended to study numerical errors due to non-reflecting boundary conditions in DGM schemes. The effects of various numerical non-reflecting boundary conditions will be treated in a unified way as either a variation in the flux formula or a variation in the underlying governing equations across a mesh interface.

Non-reflecting boundary condition is an essential part of any numerical code in computational aeroacoustics. In this paper, we will analyze three types of non-reflecting boundary conditions for DGM, namely, the characteristic non-reflecting boundary condition, finite wave model

\footnotetext{
* Professor, Senior Member AIAA

† Senior Research Scientist, Senior Member AIAA

Copyright (C) 2003 by the American Institute of Aeronautics and Astronautics, Inc. No copyright is asserted in the United States under Title 17, U.S. Code. The U.S. Government has a royalty-free license to exercise all rights under the copyright claimed herein for Governmental Purposes. All other rights are reserved by the copyright owner.
}

boundary condition and the Perfectly Matched Layer (PML) absorbing boundary condition. An earlier version of PML for the linearized Euler equation was given in split physical variables. Its implementation in the discontinuous Galerkin schemes has been demonstrated in [1]. An unsplit version has been proposed recently in [5] that eliminates numerical instabilities that can occur in the earlier version. The implementation and the discrete analysis of the unsplit version will be studied in the present paper. We will recognize the advantage of DGM in that, unlike the finite difference schemes, the absorption coefficient in the PML domain is no longer required to be continuously varying but can be increased discontinuously. Although the PML is reflectionless in its formulation in the non-discrete partial differential equations, the discretization of the equations can nonetheless cause reflections. We will first present an eigensolution analysis of the discretized PML equations in DGM. Then, the results of the eigensolution analysis will be applied to study wave reflections caused by the discretization process at the interface of the Euler and PML domains.

We will also present a discrete analysis of the characteristic non-reflecting boundary condition $[8,9]$ as well as the finite wave model boundary condition proposed in [2]. The characteristic non-reflecting boundary condition is widely used in DGM schemes because it is easy to implement due to the intrinsic upwinding features of the schemes. The finite wave model non-reflecting boundary condition proposed in [2] improves the char- 
acteristics boundary condition in that the reflection of out-going waves can be annihilated under certain conditions. The results of our discrete analysis will be compared with the theoretical reflection coefficients obtained from the non-discrete partial differential equations as well as those obtained from direct numerical simulations.

In the next section, we will first describe the discretization of the PML equation in the DGM schemes and present an eigensoultion analyses of the semi-discrete system. Since the Euler equation is a special case of the PML equation, the discrete analysis can be readily extended to the analysis of the characteristic and finite wave model non-reflecting boundary conditions in subsequent sections.

\section{Discrete analysis of PML absorbing boundary condition}

It has been shown in [5] that the PML equation is perfectly matched to the Euler equation (1). That is, in the non-discrete form, there is no reflection created at an interface between the Euler and PML domains. The effects of numerical discretization by the DGM scheme on this reflectionless property will be studied in two steps. First, we will compute the eigensolutions of the semi-discrete PML equation and study the effects of the absorbing coefficients on the wave propagation properties. Second, we will utilize the eigensolutions so formed to compute the wave reflection and transmission coefficients at an interface of discretized Euler and PML domains.

We start with the the linearized Euler equation in conservation form,

$$
\frac{\partial \mathbf{u}}{\partial t}+\nabla \cdot \mathbf{F}(\mathbf{u})=0
$$

where $\mathbf{F}(\mathbf{u})$ is the flux vector given by

$$
\mathbf{F}(\mathbf{u})=\left(\mathbf{A}_{1} \mathbf{u}, \mathbf{A}_{2} \mathbf{u}\right)
$$

in which

$$
\begin{gathered}
\mathbf{u}=\left(\begin{array}{l}
u \\
v \\
p
\end{array}\right), \mathbf{A}_{1}=\left(\begin{array}{ccc}
M & 0 & 1 \\
0 & M & 0 \\
1 & 0 & M
\end{array}\right), \\
\mathbf{A}_{2}=\left(\begin{array}{lll}
0 & 0 & 0 \\
0 & 0 & 1 \\
0 & 1 & 0
\end{array}\right)
\end{gathered}
$$

where $u$ and $v$ are velocity components (nondimensionalized by the speed of sound) in the spatial $x_{1}$ and $x_{2}$ directions respectively; $p$ is the pressure; $M$ is the Mach number of the mean flow. For conciseness, the density equation is not included here.

At non-reflecting boundaries, absorbing layers are utilized so that the waves exiting the physical domain are damped. The PML equations to be used in the absorbing domain can be written as follows [5],

$$
\begin{gathered}
\frac{\partial \mathbf{u}}{\partial t}+\nabla \cdot \mathbf{F}_{p m l}(\mathbf{u}, \mathbf{q})+\left(\sigma_{x}+\sigma_{y}\right) \mathbf{u}+\sigma_{x} \sigma_{y} \mathbf{q} \\
+\sigma_{x} \beta \mathbf{A}_{1}\left(\mathbf{u}+\sigma_{y} \mathbf{q}\right)=0,
\end{gathered}
$$

and

$$
\frac{\partial \mathbf{q}}{\partial t}=\mathbf{u}
$$

where $\mathbf{q}$ is a vector of auxiliary variables and $\beta$ in (4) is a function of the mean flow Mach number as

$$
\beta=\frac{M}{1-M^{2}}
$$

The flux vector $\mathbf{F}_{p m l}$ in (4) is of the form

$$
\mathbf{F}_{p m l}(\mathbf{u}, \mathbf{q})=\left(\mathbf{A}_{1}\left(\mathbf{u}+\sigma_{y} \mathbf{q}\right), \mathbf{A}_{2}\left(\mathbf{u}+\sigma_{x} \mathbf{q}\right)\right) .
$$

Here the absorption coefficients $\dot{\sigma}_{x}$ and $\sigma_{y}$ can be constants or functions of $x$ and $y$ respectively [5].

We consider a discontinuous Galerkin scheme for (4)-(5) in which the spatial domain is partitioned into elements denoted by $\Omega_{n m}$, where $n$ and $m$ are the element indices in the directions of $x_{1}$ and $x_{2}$ (spatial coordinates) respectively, as in Figure 1. For simplicity, we will only consider uniform rectangular elements in this paper. Triangular elements can be studied in a similar approach as we have done in [7] for the Euler equations. For each element in the PML domain, the numerical solution vectors, denoted by $\mathbf{u}_{h}^{n m}$ and $\mathbf{q}_{h}^{n m}$, are approximated by an expansions in polynomials

$$
\begin{aligned}
\mathbf{u}_{h}^{n m}(\mathbf{x}, t) & =\sum_{\ell=0}^{L} \mathbf{c}_{\ell}^{n m}(t) \phi_{\ell}^{n m}(\mathbf{x}), \\
\mathbf{q}_{h}^{n m}(\mathbf{x}, t) & =\sum_{\ell=0}^{L} \mathbf{d}_{\ell}^{n m}(t) \phi_{\ell}^{n m}(\mathbf{x}),
\end{aligned}
$$

where $\left\{\phi_{\ell}^{n m}(\mathbf{x}), \ell=0,1, . ., L\right\}$ is the set of the basis polynomials and $\mathbf{c}_{\ell}^{n m}(t)$ and $\mathbf{d}_{\ell}^{n m}(t)$ are the expansion coefficients for $\mathbf{u}_{h}^{n m}$ and $\mathbf{q}_{h}^{n m}$ respectively. A weak formulation of (4)-(5) is obtained by substituting (7)-(8) into (4)-(5) and requiring that the equations be orthogonal to the basis functions. After applying integration by parts, the semi-discrete equations for (4) and (5) are 


$$
\begin{array}{r}
\int_{\Omega_{n m}} \frac{\partial \mathbf{u}_{h}^{n m}}{\partial t} \phi_{\ell}^{n m}(\mathbf{x}) d \mathbf{x}+\int_{\Gamma_{n m}}\left(\mathbf{n} \cdot \mathbf{F}_{p m l}\right) \phi_{\ell}^{n m}(\mathbf{x}) d s \\
-\int_{\Omega_{n m}} \mathbf{F}_{p m l} \cdot \nabla \phi_{\ell}^{n m}(\mathbf{x}) d \mathbf{x}+\int_{\Omega_{n m}} \mathbf{s}_{h}^{n m} \phi_{\ell}^{n m}(\mathbf{x}) d \mathbf{x}=0,
\end{array}
$$

and

$$
\int_{\Omega_{n m}} \frac{\partial \mathbf{q}_{h}^{n m}}{\partial t} \phi_{\ell}^{n m}(\mathbf{x}) d \mathbf{x}=\int_{\Omega_{n m}} \mathbf{u}_{h}^{n m} \phi_{\ell}^{n m}(\mathbf{x}) d \mathbf{x}
$$

for $\ell=0,1, \ldots, L$, where $\Gamma_{n m}$ denotes the boundary of $\Omega_{n m}$ and $\mathbf{n}=\left(n_{1}, n_{2}\right)$ is its outward normal. Here $\mathbf{s}_{h}^{n m}$ in (9) represents the non-differential terms in (4):

$\mathbf{s}_{h}^{n m}=\left(\sigma_{x}+\sigma_{y}\right) \mathbf{u}_{h}^{n m}+\sigma_{x} \sigma_{y} \mathbf{q}_{h}^{n m}+\sigma_{x} \beta \mathbf{A}_{1}\left(\mathbf{u}_{h}^{n m}+\sigma_{y} \mathbf{q}_{h}^{n m}\right)$.

Equations (9) and (10) will result in a system of ordinary differential equations for the expansion coefficients $\mathbf{c}_{\ell}^{n m}(t)$ and $\mathbf{d}_{\ell}^{n m}(t)$.

For the PML flux vector given in (6), the normal flux at the edge of an element is

$$
\mathbf{n} \cdot \mathbf{F}_{p m l}=\left(n_{1} \mathbf{A}_{1}+n_{2} \mathbf{A}_{2}\right) \mathbf{u}+\left(\sigma_{y} n_{1} \mathbf{A}_{1}+\sigma_{x} n_{2} \mathbf{A}_{2}\right) \mathbf{q}
$$

For convenience of discussion, we denote the normal flux in the original Euler equation by

$$
\mathbf{n}: \mathbf{F}=\left(n_{1} \mathbf{A}_{1}+n_{2} \mathbf{A}_{2}\right) \mathbf{u}=\mathbf{A}_{n} \mathbf{u},
$$

which is the same as the first term in $\mathbf{n} \cdot \mathbf{F}_{p m l}$ above. Further, it is easy to verify that the second term in (11) can be simplified as

$\left(\sigma_{y} n_{1} \mathbf{A}_{1}+\sigma_{x} n_{2} \mathbf{A}_{2}\right) \mathbf{q}= \begin{cases}\sigma_{y} \mathbf{A}_{n} \mathbf{q} & \text { vertical edges }\left(n_{2}=0\right) \\ \sigma_{x} \mathbf{A}_{n} \mathbf{q} & \text { horizontal edges }\left(n_{1}=0\right)\end{cases}$

This leads to the following more compact flux expression on the edges

$$
\mathbf{n} \cdot \mathbf{F}_{p m l}=\mathbf{A}_{n}(\mathbf{u}+\sigma \mathbf{q})
$$

where $\sigma$ is either $\sigma_{x}$ or $\sigma_{y}$ according to (13).

Since we do not require that the numerical solution be continuous across the interface of any two elements, the normal flux $\mathbf{n} \cdot \mathbf{F}_{p m l}$ appearing in the edge integral in (9) is not uniquely determined. To complete the formulation, a numerical flux formula needs to be specified. As in references [6] and [7], we will consider two commonly used flux formulas, namely, the characteristics splitting flux formula and the Lax-Friedrich flux formula. For convenience of discussion, let $\mathbf{u}_{h}^{+}, \mathbf{q}_{h}^{+}$and $\mathbf{u}_{h}^{-}, \mathbf{q}_{h}^{-}$denote the values of $\mathbf{u}_{h}, \mathbf{q}_{h}$ on the edge of an element evaluated using the solutions inside and outside of that element respectively. Then both flux formulas can be conveniently written as follows

$$
\mathbf{n} \cdot \mathbf{F}_{p m l}=\mathbf{A}_{+}\left(\mathbf{u}_{h}^{+}+\sigma \mathbf{q}_{h}^{+}\right)+\mathbf{A}_{-}\left(\mathbf{u}_{h}^{-}+\sigma \mathbf{q}_{h}^{-}\right)
$$

where

$$
\mathbf{A}_{+}=\frac{1}{2}\left(\mathbf{A}_{n}+\left|\mathbf{A}_{n}\right|\right) \text { and } \mathbf{A}_{-}=\frac{1}{2}\left(\mathbf{A}_{n}-\left|\mathbf{A}_{n}\right|\right)
$$

for the characteristics splitting flux formula and

$$
\mathbf{A}_{+}=\frac{1}{2}\left(\mathbf{A}_{n}+\left|a_{\max }\right| \mathbf{I}\right) \text { and } \mathbf{A}_{-}=\frac{1}{2}\left(\mathbf{A}_{n}-\left|a_{\max }\right| \mathbf{I}\right)
$$

for the Lax-Friedrichs flux formula, where $a_{\max }$ is the largest (in magnitude) eigenvalue of $\mathbf{A}_{n}$.

In our analysis, we assume that the same flux formula is used through out the computational domain.

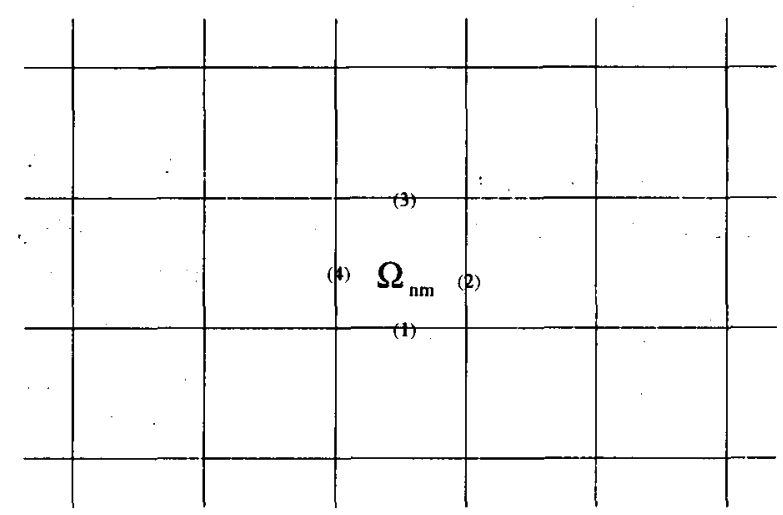

Figure 1. A schematic of partitioning of the computational domain in quadrilateral elements. Here, $n$ and $m$ are the indices of the element and the number inside the parentheses indicates the ordering of the element edges.

We order and denote the four edges of $\Omega_{n m}$ as $\Gamma_{n m}^{(i)}, i=$ $1,2,3,4$, as shown in Figure 1. Using (15) for the normal flux, the weak formulation (9) can now be written in detail as

$$
\begin{gathered}
\int_{\Omega_{n m}} \phi_{\ell}(\mathbf{x}) \frac{\partial \mathbf{u}_{h}^{n m}}{\partial t} d \mathbf{x} \\
+\int_{\Gamma_{n m}^{(1)}} \phi_{\ell}(\mathbf{x})\left[\mathbf{A}_{+}^{(1)} \mathbf{u}_{x}^{n m}+\mathbf{A}_{-}^{(1)} \mathbf{u}_{x}^{n m-1}\right] d s \\
+\int_{\Gamma_{n m}^{(2)}} \phi_{\ell}(\mathbf{x})\left[\mathbf{A}_{+}^{(2)} \mathbf{u}_{y}^{n m}+\mathbf{A}_{-}^{(2)} \mathbf{u}_{y}^{n+1 m}\right] d s
\end{gathered}
$$




$$
\begin{gathered}
+\int_{\Gamma_{n m}^{(3)}} \phi_{\ell}(\mathbf{x})\left[\mathbf{A}_{+}^{(3)} \mathbf{u}_{x}^{n m}+\mathbf{A}_{-}^{(3)} \mathbf{u}_{x}^{n m+1}\right] d s \\
+\int_{\Gamma_{n m}^{(4)}} \phi_{\ell}(\mathbf{x})\left[\mathbf{A}_{+}^{(4)} \mathbf{u}_{y}^{n m}+\mathbf{A}_{-}^{(4)} \mathbf{u}_{y}^{n-1 m}\right] d s \\
-\int_{\Omega_{n m}} \frac{d \phi_{\ell}}{d x} \mathbf{A}_{1} \mathbf{u}_{y}^{n m} d \mathbf{x}-\int_{\Omega_{n m}} \frac{d \phi_{\ell}}{d y} \mathbf{A}_{2} \mathbf{u}_{x}^{n m} d \mathbf{x} \\
+\int_{\Omega_{n m}} \mathbf{s}_{h}^{n m} \phi_{\ell^{\prime}}^{n m}(\mathbf{x}) d \mathbf{x}=0
\end{gathered}
$$

where we have used notation

$$
\mathbf{u}_{x, y}^{n m} \equiv \mathbf{u}_{h}^{n m}+\sigma_{x, y} \mathbf{q}_{h}^{n m} .
$$

The superscripts (1) - (4) in equation (18) indicate the specific edge of the element for the integrals as shown in Figure 1.

\section{Eigensolutions of the discrete system}

To look for wave-like solutions to (18), we assume periodicity in time with a frequency $\omega$ and let

$$
\begin{aligned}
& \mathbf{u}_{h}^{n m}(\xi, \eta, t)=e^{-i \omega t} \sum_{\ell=0}^{L} \hat{\mathbf{c}}_{\ell}^{n m} \phi_{\ell}(\xi, \eta), \\
& \mathbf{q}_{h}^{n m}(\xi, \eta, t)=e^{-i \omega t} \sum_{\ell=0}^{L} \hat{\mathbf{d}}_{\ell}^{n m} \phi_{\ell}(\xi, \eta),
\end{aligned}
$$

where $\xi$ and $\eta$ are local coordinates. The coefficients $\hat{\mathbf{c}}_{\ell}^{n m}$ and $\hat{\mathbf{q}}_{\ell}^{n m}$ are now independent of $t$.

For convenience of discussion, define vectors

$$
\hat{\mathbf{C}}^{n m}=\left(\begin{array}{c}
\hat{\mathbf{c}}_{1}^{n m} \\
\hat{\mathbf{c}}_{2}^{n m} \\
: \\
\hat{\mathbf{c}}_{L}^{n m}
\end{array}\right), \hat{\mathbf{D}}^{n m}=\left(\begin{array}{c}
\hat{\mathbf{d}}_{1}^{n m} \\
\hat{\mathbf{d}}_{2}^{n m} \\
\vdots \\
\hat{\mathbf{d}}_{L}^{n m}
\end{array}\right)
$$

and

$$
\mathbf{P}(\xi, \eta)=\left(\begin{array}{c}
\phi_{1}(\xi, \eta) \\
\phi_{2}(\xi, \eta) \\
\vdots \\
\phi_{L}(\xi, \eta)
\end{array}\right)
$$

Then, using the Kronecker product $\otimes$ (see reference [6] for definition and its properties), the solution in (20) and (21) can be expressed as follows,

$$
\begin{aligned}
& \mathbf{u}_{h}^{n m}(\xi, \eta, t)=e^{-i \omega t}\left(\mathbf{P}^{T} \otimes \mathbf{I}\right) \hat{\mathbf{C}}^{n m}, \\
& \mathbf{q}_{h}^{n m}(\xi, \eta, t)=e^{-i \omega t}\left(\mathbf{P}^{T} \otimes \mathbf{I}\right) \hat{\mathbf{D}}^{n m} .
\end{aligned}
$$

By substituting (20) and (21), or their tensor forms (22) and (23), into the semi-discrete PML equations (18) and (10), we get a linear system for the expansion coeffcients $\hat{\mathbf{C}}^{n m}$ and $\hat{\mathbf{D}}^{n m}$ as follows:

$$
\begin{gathered}
-i \omega(\mathbf{Q} \otimes \mathbf{I}) \hat{\mathbf{C}}^{n m}+\left(\mathbf{B}^{(1)} \otimes \mathbf{A}_{+}^{(1)}\right) \hat{\mathbf{C}}_{x}^{n m}+\left(\mathbf{B}^{\prime(1)} \otimes \mathbf{A}_{-}^{(1)}\right) \hat{\mathbf{C}}_{x}{ }^{n m-1} \\
+\left(\mathbf{B}^{(2)} \otimes \mathbf{A}_{+}^{(2)}\right) \hat{\mathbf{C}}_{y}^{n m}+\left(\mathbf{B}^{\prime(2)} \otimes \mathbf{A}_{-}^{(2)}\right) \hat{\mathbf{C}}_{y}^{n+1 m} \\
+\left(\mathbf{B}^{(3)} \otimes \mathbf{A}_{+}^{(3)}\right) \hat{\mathbf{C}}_{x}^{n m}+\left(\mathbf{B}^{\prime(3)} \otimes \mathbf{A}_{-}^{(3)}\right) \hat{\mathbf{C}}_{x}{ }^{n m+1} \\
+\left(\mathbf{B}^{(4)} \otimes \mathbf{A}_{+}^{(4)}\right) \hat{\mathbf{C}}_{y}^{n m}+\left(\mathbf{B}^{\prime(4)} \otimes \mathbf{A}_{-}^{(4)}\right) \hat{\mathbf{C}}_{y}{ }^{n-1 m} \\
-\left(\mathbf{Q}_{x} \otimes \mathbf{A}_{1}\right) \hat{\mathbf{C}}_{y}{ }^{n m}-\left(\mathbf{Q}_{y} \otimes \mathbf{A}_{2}\right) \hat{\mathbf{C}}_{x}{ }^{n m} \\
+(\mathbf{Q} \otimes \mathbf{I})\left[\left(\sigma_{x}+\sigma_{y}\right) \hat{\mathbf{C}}^{n m}+\sigma_{x} \sigma_{y} \hat{\mathbf{D}}^{n m}\right]+\sigma_{x} \beta\left(\mathbf{Q} \otimes \mathbf{A}_{1}\right) \hat{\mathbf{C}}_{y}^{n m}=0 \\
\end{gathered}
$$

and

where

$$
-i \omega(\mathbf{Q} \otimes \mathbf{I}) \hat{\mathbf{D}}^{n m}=(\mathbf{Q} \otimes \mathbf{I}) \hat{\mathbf{C}}^{n m}
$$

$$
\hat{\mathbf{C}}_{x, y}^{n m}=\hat{\mathbf{C}}^{n m}+\sigma_{x, y} \hat{\mathbf{D}}^{n m}
$$

and $\mathbf{Q}$ is the "mass matrix", $\mathbf{Q}_{x}$ and $\mathbf{Q}_{y}$ are the "stiffness" matrices, and $\mathbf{B}^{(i)}$ and $\mathbf{B}^{\prime(i)}$ are the "edge" matrices resulted from the edge integrals in (18) involving solutions inside and outside of the element respectively. The detailed definitions of these matrices are given in the Appendix (A1).

By (25), we easily get

$$
\hat{\mathbf{D}}^{n m}=\frac{i}{\omega} \hat{\mathbf{C}}^{n m} \text {. }
$$

As a result, equation (24) can be written in $\hat{\mathbf{C}}^{n m}$ only as

$$
\begin{array}{r}
-i \omega\left(1+\frac{i \sigma_{x}}{\omega}\right)\left(1+\frac{i \sigma_{y}}{\omega}\right)(\mathbf{Q} \otimes \mathbf{I}) \hat{\mathbf{C}}^{n m}+\mathbf{H}^{(0)} \hat{\mathbf{C}}^{n m} \\
+\left(1+\frac{i \sigma_{x}}{\omega}\right) \mathbf{H}^{(1)} \hat{\mathbf{C}}^{n m-1}+\left(1+\frac{i \sigma_{y}}{\omega}\right) \mathbf{H}^{(2)} \hat{\mathbf{C}}^{n+1 m} \\
+\left(1+\frac{i \sigma_{x}}{\omega}\right) \mathbf{H}^{(3)} \hat{\mathbf{C}}^{n m+1}+\left(1+\frac{i \sigma_{y}}{\omega}\right) \mathbf{H}^{(4)} \hat{\mathbf{C}}^{n-1 m}=0 .
\end{array}
$$

The matrices $\mathbf{H}^{(0)-(4)}$ can be obtained by directly comparing (27) with (24) and will not be given explicitly here. 
For a uniform distribution of elements, the coefficient matrices in (27) are independent of the element indices $n$ and $m$. We seek solutions of the form

$$
\hat{\mathbf{C}}^{n m}=\lambda_{1}^{n} \lambda_{2}^{m} \tilde{\mathbf{C}}
$$

where $\lambda_{1}$ and $\lambda_{2}$ are related to wavenumbers $k_{1}$ and $k_{2}$ in the $x_{1}$ and $x_{2}$ directions, respectively, as follows

$$
\lambda_{1}=e^{i k_{1} h_{1}}, \quad \lambda_{2}=e^{i k_{2} h_{2}}
$$

where $h_{1}$ and $h_{2}$ are the spatial dimensions of elements in $x_{1}$ and $x_{2}$ directions respectively. An eigenvalue problem for $\tilde{\mathbf{C}}$ is formed by substituting (28) into (27) which yields immediately

$$
\begin{array}{r}
-i \omega\left(1+\frac{i \sigma_{x}}{\omega}\right)\left(1+\frac{i \sigma_{y}}{\omega}\right)(\mathbf{Q} \otimes \mathbf{I}) \tilde{\mathbf{C}}+\mathbf{H}^{(0)} \tilde{\mathbf{C}} \\
+\left(1+\frac{i \sigma_{x}}{\omega}\right) \frac{1}{\lambda_{2}} \mathbf{H}^{(1)} \tilde{\mathbf{C}}+\lambda_{\mathbf{1}}\left(1+\frac{i \sigma_{y}}{\omega}\right) \mathbf{H}^{(2)} \tilde{\mathbf{C}} \\
+\left(1+\frac{i \sigma_{x}}{\omega}\right) \lambda_{2} \mathbf{H}^{(3)} \tilde{\mathbf{C}}+\frac{1}{\lambda_{1}}\left(1+\frac{i \sigma_{y}}{\omega}\right) \mathbf{H}^{(4)} \tilde{\mathbf{C}}=0 .
\end{array}
$$

To study the spatially propagating waves, we solve for $\lambda_{1}$ and $\tilde{\mathbf{C}}$ as the eigenvalue and eigenvector from (30) for given $\omega$, the frequency, and $k_{2}$, the wavenumber in $x_{2}$ direction. The corresponding discrete wave number $k_{1}^{*}$ of the eigensolution can be found according to (29):

$$
k_{1}^{*} h_{1}=-i \ln \left(\lambda_{1}\right) .
$$

It is well known that, for any given values of $\omega$ and $k_{2}$, the Euler equation (1) supports two acoustic waves given by the dispersion relation

$$
\left(\omega-M k_{1}\right)^{2}-k_{1}^{2}-k_{2}^{2}=0
$$

and one vorticity wave given by

$$
\omega-M k_{1}=0 .
$$

The acoustic waves can be conveniently expressed in the given frequency $\omega$ and a wave angle $\phi$ as

$$
k_{1}=\frac{\omega \cos \phi}{1+M \cos \phi}, \quad k_{2}=\frac{\omega \sin \phi}{1+M \cos \phi}
$$

To illustrate typical eigensolutions of (30), we solve the eigenvalue problem with square elements $h_{1}=h_{2}=h$ and the order of basis functions $p=2$. In Figure 2, we show the discrete wavenumber $k_{1}^{*}$ corresponding to the right-traveling acoustic mode obtained as the eigenvalue of (30). Plotted in Figure 2a are the ratio of the real part of $k_{1}^{*}$ and the exact $k_{1}$ in (33) as a function of nondimensionalized frequency $\omega h$. In Figure $2 \mathrm{~b}$, we show the imaginary part of $k_{1}^{*}$. The value of $k_{2}$, hence the value of $\lambda_{2}$ in (30), is given according to (33) and (29) with $\phi=\pi / 6$. The Mach number of the flow is $M=0.5$. For simplicity, $\sigma_{y}=0$ in all the calculations. The solid line in both figures represents the result for $\sigma_{x}=0$, i.e., that of the discretized Euler equation, and dashed lines represent that of the PML equation with non-zero $\sigma_{x}$ as indicated. Figure 2 illustrates the effects of the absorption coefficient on the discrete wavenumber in the PML domain. Figure 2a indicates that an increase in the absorption coefficient has very little effect on the real part of $k_{1}^{*}$ for waves within the resolved range in non-dimensionalized frequency $\omega h<1$, see reference [7]. The imaginary part of $k_{1}^{*}$, however, has an increase that is proportional to the increase in the absorption coefficient, indicating exponentional absorption of the wave. Furthermore, the damping rate remains the same for all frequencies within the resolved range $\omega h<1$. This means that the absorption rate for the acoustic wave will be independent of the wave frequency.

In Figure 3, we show the dependence of PML damping rate as a function of the wave angle for a given wave frequency $\omega h=0.5$. In general, the damping rate reduces as the wave angle $\theta$ increases. This is consistent with the theoretical prediction of the non-discrete PML equation [5].

Aside from the physical modes, there are also nonphysical eigensolutions, or spurious modes, for (30). The number of spurious modes depends on the order of the basis functions and the flux formula being used. It can be shown that there are $p$ spurious modes for each physical wave of the non-discrete partial differential equation when the exact characteristics splitting is used in the flux formula. The number of the spurious mode will be increased to be $2 p+1$ when Lax-Friedrichs flux formula is used. The non-physical waves are irregular and highly damped $[6,7]$.

\section{Reflection and transmission at an interface of Euler and PML domains}

Although the PML is reflectionless in the non-discrete limit, discretization of the partial differential equations may cause numerical reflections at the interface of the Euler and PML domains. We will now analyze the numerical reflection error due to the discretization process.

Without loss of generality, suppose that the elements in the Euler domain have indices $n \leq 0$ and those in the PML domain have indices $n \geq 1$, as shown in Figure 4. We will introduce an out-going wave (right-traveling 

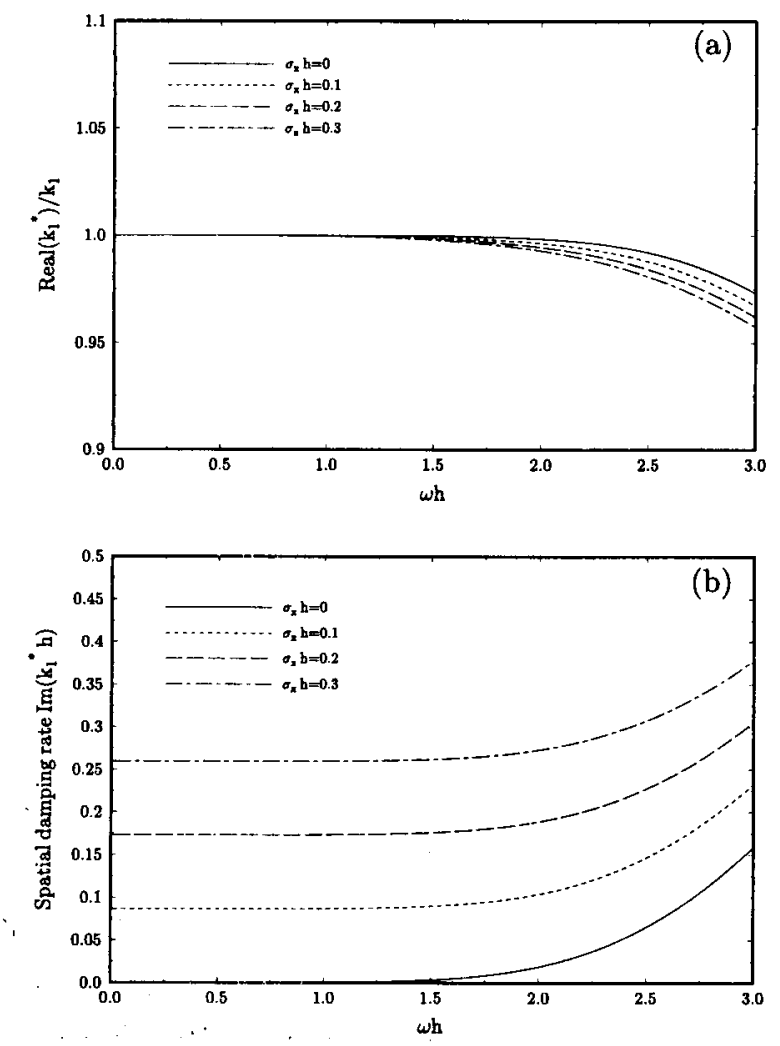

Figure 2. Real and imaginary parts of the numerical wavenumber $k_{1}^{*}$ of the physical mode of (30) as functions of wave frequency wh. $h_{1}=h_{2}=h, M=0.5, p=2$. $\sigma_{y}=0$.

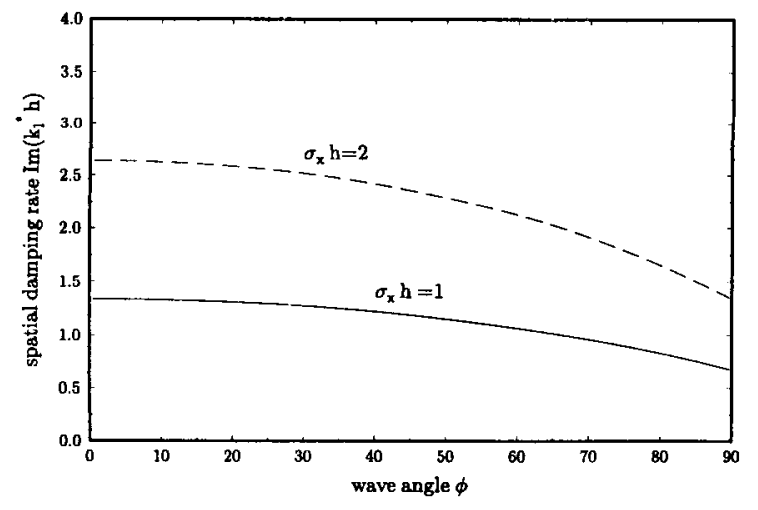

Figure 3. Normalized numerical wave speed and spatial damping rate of the physical mode as functions of wave frequency. $\theta=\pi / 6, p=2, h_{1}=h_{2}=1 . \quad \sigma_{y}=0$. $\omega h=0.5$.

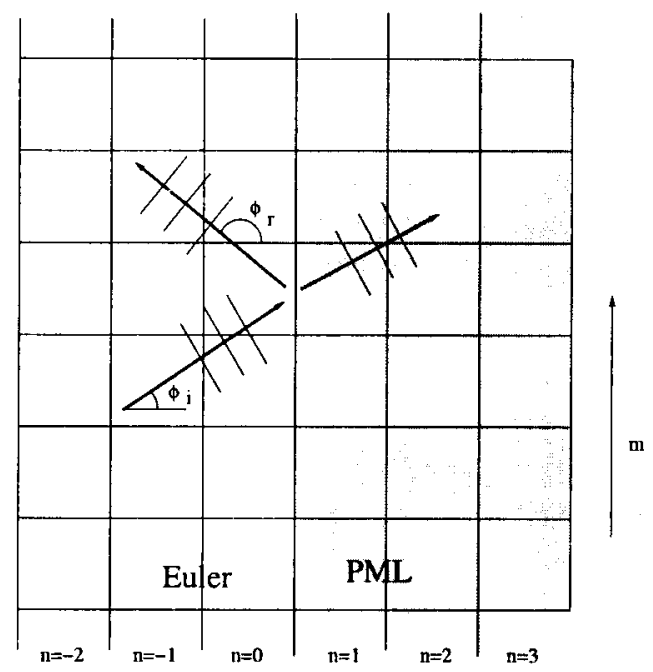

Figure 4. A schematic of wave reflection and transmission at an interface of Euler and PML domains. The $\phi_{i}$ is the wave angle of the out-going wave.

in Figure 4) in the Euler domain and compute the reflected and transmitted waves in the Euler and PML domains respectively. For an interface shown in Figure 4 , the reflected waves will include all the left-traveling eigensolutions of the discrete Euler equation and the transmitted waves will include all the right-traveling eigensolutions of the discrete PML equation. Both the physical and non-physical modes have to be considered. Specifically, we assume the solution in each half domain as

for $n \leq 0$ :

$$
\hat{\mathbf{C}}_{E u l e r}^{n m}=\mathbf{V}_{W^{R}}^{n m}+\beta_{W^{L}} \mathbf{V}_{W^{L}}^{n m}+\sum_{j} \beta_{E_{j}^{L}} \mathbf{V}_{E_{j}^{L}}^{n m},
$$

for $n \geq 1$ :

$$
\hat{\mathbf{C}}_{p m l}^{n m}=\beta_{W^{R}}^{p m l} \mathbf{V}_{W^{R}}^{n m}+\sum_{j} \beta_{E_{j}^{R}}^{p m l} \mathbf{V}_{E_{j}^{R}}^{n m}
$$

where subscripts $W^{R}$ and $W^{L}$ denote the physical right and left traveling waves respectively, and $E_{j}^{R}$ and $E_{j}^{L}$ denote the non-physical right and left traveling spurious wave modes respectively. Here the $\mathbf{V}$ vectors are the corresponding eigenvectors of the semi-discrete equations. In (34) and (35), the out-going wave, $\mathbf{V}_{W^{L}}^{n m}$, has an amplitude of unity, and $\beta_{W^{L}}$ and $\beta_{E_{i}^{L}}$ are the reflection coefficients and $\beta_{W^{R}}$ and $\beta_{E_{j}^{R}}$ are the transmission coefficients.

The coupling of the two solutions occurs at elements immediately on either side of the interface, namely, the 
elements with indices $n=0$ and $n=1$. The semidiscrete equation for the Euler domain is that given in (27) with $\sigma_{x}=\sigma_{y}=0$. For the PML domain shown in Figure 4, we have $\sigma_{y}=0$ but $\sigma_{x} \neq 0$. Thus, we have

for $n=0$ :

$$
\begin{gathered}
-i \omega(\mathbf{Q} \otimes \mathbf{I}) \hat{\mathbf{C}}_{E u l e r}^{0, m}+\mathbf{H}^{(0)} \hat{\mathbf{C}}_{E u l e r}^{0, m}+\mathbf{H}^{(1)} \hat{\mathbf{C}}_{E u l e r}^{0, m-1} \\
+\mathbf{H}^{(2)} \hat{\mathbf{C}}_{p m l}^{1, m}+\mathbf{H}^{(3)} \hat{\mathbf{C}}_{E u l e r}^{0, m+1}+\mathbf{H}^{(4)} \hat{\mathbf{C}}_{E u l e r}^{-1, m}=0
\end{gathered}
$$

for $n=1$ :

$$
\begin{gathered}
-i \omega\left(1+\frac{i \sigma_{x}}{\omega}\right)(\mathbf{Q} \otimes \mathbf{I}) \hat{\mathbf{C}}_{p m l}^{1, m}+\mathbf{H}^{(0)} \hat{\mathbf{C}}_{p m l}^{1, m} \\
+\left(1+\frac{i \sigma_{x}}{\omega}\right) \mathbf{H}^{(1)} \hat{\mathbf{C}}_{p m l}^{1, m-1}+\mathbf{H}^{(2)} \hat{\mathbf{C}}_{p m l}^{2, m} \\
+\left(1+\frac{i \sigma_{x}}{\omega}\right) \mathbf{H}^{(3)} \hat{\mathbf{C}}_{p m l}^{1, m+1}+\mathbf{H}^{(4)} \hat{\mathbf{C}}_{E u l e r}^{0, m}=0
\end{gathered}
$$

Here, the absorption coefficient $\sigma_{x}$ is assumed to be constant for all the elements in the PML domain.

Equations (36) and (37) can be further simplified by the fact that $\mathbf{C}_{E u l e r}^{n m}$ and $\mathbf{C}_{p m l}^{n m}$ are formed by the eigenvectors. As a result, we get the following more compact matching conditions [7],

$$
\begin{aligned}
& \mathbf{H}^{(2)} \hat{\mathbf{C}}_{\text {Euler }}^{1, m}=\mathbf{H}^{(2)} \hat{\mathbf{C}}_{p m l}^{1, m}, \\
& \mathbf{H}^{(4)} \hat{\mathbf{C}}_{E \text { uler }}^{0, m}=\mathbf{H}^{(4)} \hat{\mathbf{C}}_{p m l}^{0, m}
\end{aligned}
$$

By substituting (34) and (35) into (38) and (39), we can find the numerical reflection coefficients at the interface.

As an example, we consider an out-going acoustic wave with a wave angle $\phi_{i}=\pi / 6$. The order of the basis functions used is $p=2$. For this order, when the exact characteristic splitting formula (16) is used, the reflected waves in the Euler domain include the left-traveling acoustic mode, denoted by $W^{L}$, and two non-physical spurious modes, denoted by $E_{1}^{L}$ and $E_{2}^{L}$. Figure 5 shows the reflection coefficients of the physical ( $W^{L}$, in circles) and non-physical ( $E_{1}^{L}, E_{2}^{L}$, in diamonds) modes as functions of the non-dimensional wave frequency $\omega h$. As the value of $\omega h$ decreases, i.e., when the frequency of the wave is reduced (hence its wavelength is increased) or the size of the element is decreased, the reflection at the interface caused by numerical discretization also decreases. More significantly, the reflection coefficient for the physical mode is decreasing at a higher order than that of the non-physical modes. Since the non-physical modes are highly damped by the numerical dissipation, Figure 5 indicates that the reflections occurred at the

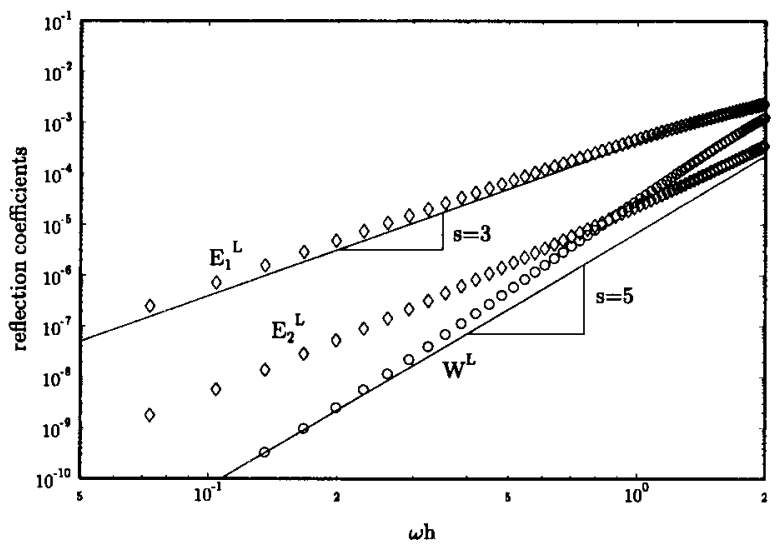

Figure 5. Reflection coefficients of the physical $\left(W^{L}\right)$ and non-physical $\left(E_{1}^{L}, E_{2}^{L}\right)$ modes with basis function order $p=2$. $M=0.5, \sigma_{x}=1$. $s$ indicates slope of solid line. The out-going acoustic wave angle $\phi_{i}=\pi / 6$. $h_{1}=h_{2}=h$.

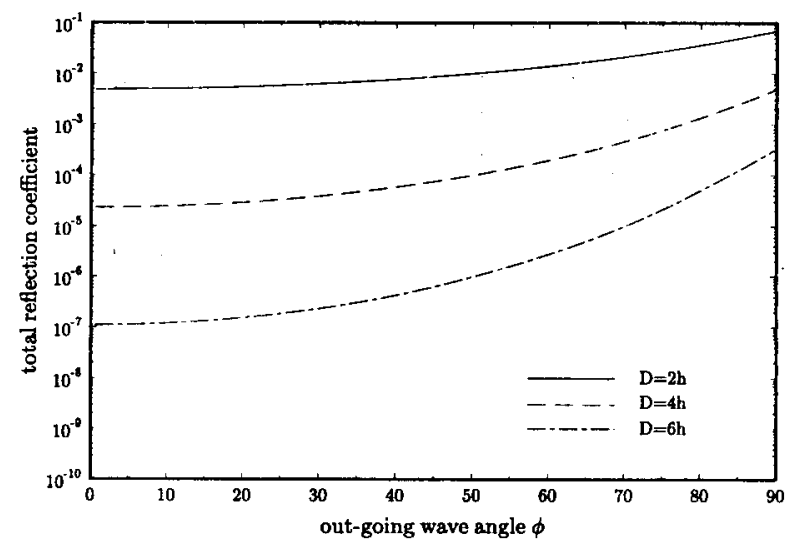

Figure 6. Reflection coefficient of the physical mode as a function of wave angle. The width of the PML domain is D. $p=2 . M=0.5, \sigma_{x} h=1$. $\omega h=0.5$.

interface will likely have only a local effect and will not be propagated globally to the computational domain. We note that when the Lax-Friedrichs flux formula was used, the number of reflected non-physical modes was increased. However, trends similar to those of Figure 5 were obtained.

The transmitted waves are damped exponentially inside the PML domain, at a rate that is determined by the imaginary part of the numerical wavenumber $k_{1}^{*}$. In practical computations, however, the PML domain is of finite width and wave reflection can occur when 
the PML domain is truncated. The waves reflected at the end of the PML domain will be damped again when traveling back through the PML domain before re-entering the Euler domain. The magnitude of the wave that re-enters the Euler domain relative to that of the initial out-going wave will be referred to the total reflection coefficient of the PML domain. Obviously, the total reflection coefficient depends on the width of the PML domain as well as the value of the absorption coefficient used. In Figure 6, we show the total reflection coefficient of the physical mode as a function of the out-going wave angle when the width of the PML domain is 2,4 and 6 elements. The value of the absorption coefficient $\sigma_{x} h$ is unity. Here, we have assumed complete reflection at the end of the PML domain. The reflection can be further reduced, in fact, by using other type of non-reflecting boundary condition to terminate the PML domain itself.

\section{Characteristic Non-reflecting Boundary Condition}

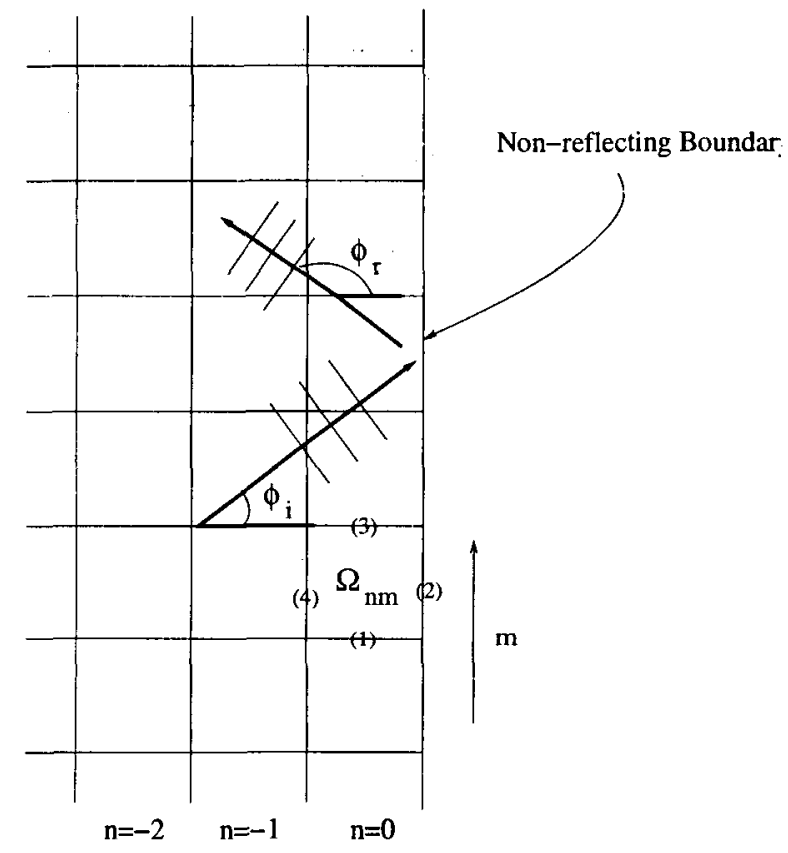

Figure 7. Schematic of elements at the non-reflecting boundary. The elements at the boundary have index $n=$ 0 . (1)-(4) indicates the order of the edges of element $\Omega_{n m}$.

We will now study the characteristic non-reflecting boundary condition applied to the discontinuous Galerkin schemes. The implementation of the boundary condition is relatively simple by using characteristics splitting flux formula (16) at the boundary elements. The non-reflecting boundary condition is imposed by eliminating the exterior flux contribution in the edge integrals along the non-reffecting boundary $[8,9]$.

Consider a non-reflecting boundary shown in Figure 7, where the boundary elements have index $n=0$. The semi-discrete equation for the Euler domain is the same as those given in (18) or (24) for the PML domain with $\sigma_{x}=\sigma_{y}=0$. For elements at the non-reflecting boundary with index $n=0$, the edge integral along the non-reflecting boundary (i.e., side (2)) in equation (18),

$$
\int_{\Gamma_{0, m}^{(2)}} \phi_{\ell}(\mathbf{x})\left[\mathbf{A}_{+}^{(2)} \mathbf{u}_{h}^{0, m}+\mathbf{A}_{-}^{(2)} \mathbf{u}_{h}^{1, m}\right] d s
$$

is replaced by

$$
\int_{\Gamma_{0, m}^{(2)}} \phi_{\ell}(\mathbf{x}) \hat{\mathbf{A}}_{+}^{(2)} \mathbf{u}_{h}^{0, m} d s
$$

where $\hat{\mathbf{A}}_{+}^{(2)}$ is the flux matrix from the characteristics splitting formula given in (16), i.e.,

$$
\hat{\mathbf{A}}_{+}^{(2)}=\frac{1}{2}\left(\mathbf{A}_{n}^{(2)}+\left|\mathbf{A}_{n}^{(2)}\right|\right)
$$

We note that when the Lax-Friedrichs flux formula is used in the interior elements, $\hat{\mathbf{A}}_{+}^{(2)}$ differs from $\mathbf{A}_{+}^{(2)}$ that is used in the eigensolution analysis of (24). They are of course the same when the characteristics splitting flux formula is used in the interior domain. The total reflection error can be further reduced by increasing the width of the PML domain.

In terms of the solution expansion coefficient vector $\hat{\mathbf{C}}^{n m}$, the characteristic non-reflecting boundary condition leads to the following equation for the elements at the boundary,

$$
\left(\mathbf{B}^{(2)} \otimes \mathbf{A}_{+}^{(2)}\right) \hat{\mathbf{C}}^{0, m}+\left(\mathbf{B}^{\prime(2)} \otimes \mathbf{A}_{-}^{(2)}\right) \hat{\mathbf{C}}^{1, m}=\left(\mathbf{B}^{(2)} \otimes \hat{\mathbf{A}}_{+}^{(2)}\right) \hat{\mathbf{C}}^{0, m} .
$$

By considering a numerical solution of the form similar to (34) for $\hat{\mathbf{C}}^{n, m}$ in (40) that consists of an out-going physical wave mode and reflected acoustic and other non-physical modes, we can compute the reflection coefficients using (40). In Figure 8, we show the magnitudes of reflected physical (acoustic) and non-physical wave modes in response to an out-going (right-traveling) acoustic wave that has the angle $\phi_{i}=\pi / 6$. The order of the basis functions used is $p=2$ and the characteristics splitting flux formula is used through out the computational domain. As the wave frequency $\omega$ or the mesh 


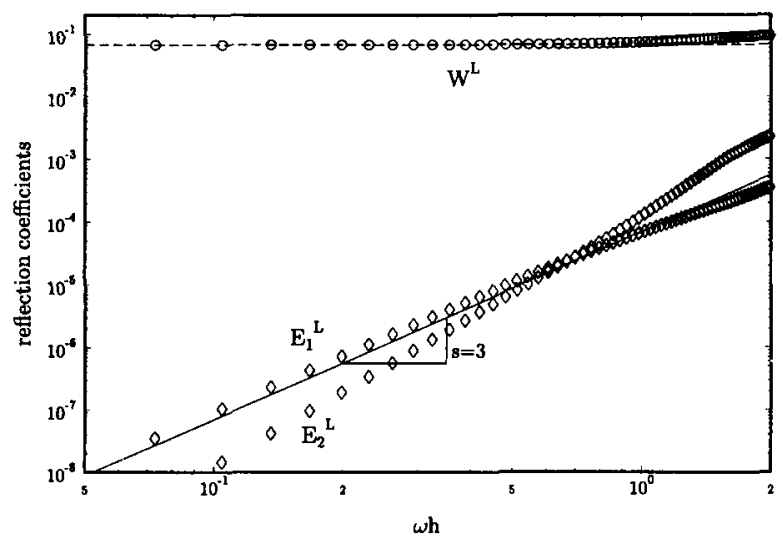

Figure 8. Reflection coefficient of the characteristics boundary condition. $W^{L}$ is the physical mode (circles) and $E_{1}^{L}$ and $E_{2}^{L}$ are non-physical modes (diamonds). $M=0.5$. Dashed line is the prediction according to the partial differential equation. $s$ indicates the slope of the solid line.

size $h$ decreases, the magnitudes of the reflected nonphysical modes (in diamond symbols) are reduced and approach to zero in the limit. However, the magnitude of the reflected acoustic mode (in circles) is approaching a non-zero limit predicted by the non-discrete partial differential equation model given in the Appendix. This is in contrast to the case of PML boundary condition shown in Figure 5 where the reflected physical mode goes to zero in the limit. It indicates that the reflection error can not be eliminated by mesh refinements for the characteristic non-reflecting boundary condition. Since a well-resolved physical mode has very small numerical damping [7], the reflection error can have a global effect on the numerical solution.

In Figure 9, we show the magnitude of the reflected physical mode (left-traveling) relative to an out-going (right-traveling) acoustic wave. Plotted are the reflection coefficients as functions of $\phi_{i}$, the out-going wave angle, for the mean flow Mach number $M=0$ and 0.5. At the resolved frequency $\omega h=0.5$, the numerical reflection coefficient coincide with that predicted by the non-discrete model shown in dashed lines. Clearly, the characteristic boundary condition is most effective when the wave angle is close to zero.

\section{Finite Wave Model}

As we have seen in the previous section, the characteristic non-reflecting boundary condition works best when the out-going wave angle $\phi_{i}$ is zero or close to zero,

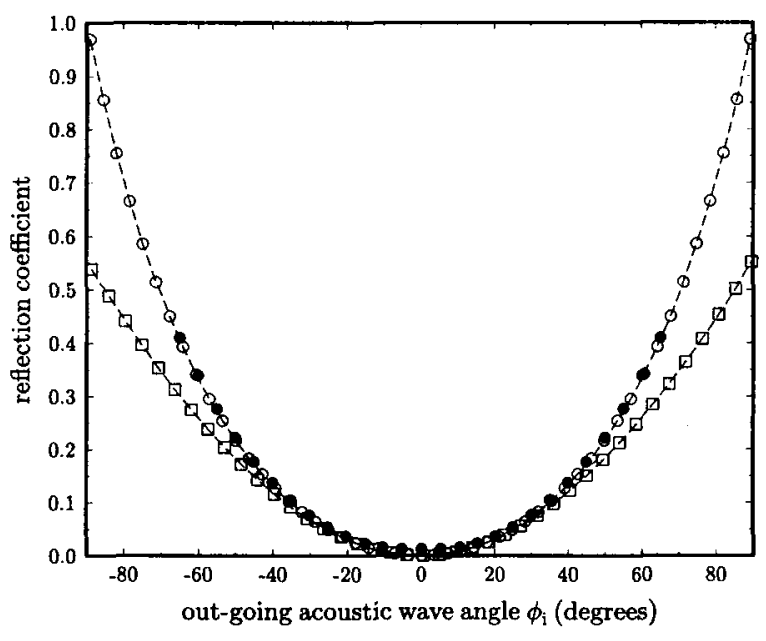

Figure 9. Reflection coefficient of the characteristics boundary condition. Circle: $M=0$; square: $M=0.5$. $\omega h=0.5$. Open symbols are the results of discrete analysis and closed symbols are results from direct numerical simulation. Dashed line is the prediction according to the solution of partial differential equations.

i.e., when the direction of wave propagation is nearly normal to the boundary. The reflection coefficient increases significantly when $\phi_{i}$ deviates from zero. Several improvements on the characteristic boundary condition have been proposed in the literature [4]. One approach is the finite wave model given in [2].

In the finite wave model, an assumption is made on the out-going wave angle and, accordingly, the solution at the boundary is constructed from the characteristic decomposition associated with that particular propagation direction. Specifically, the edge integral along the non-reflecting boundary in the semi-discrete equation (18) (with $\sigma_{x}=\sigma_{y}=0$ ) for elements with index $n=0$,

$$
\int_{\Gamma_{0, m}^{(2)}} \phi_{\ell}(\mathbf{x})\left[\mathbf{A}_{+}^{(2)} \mathbf{u}_{h}^{0, m}+\mathbf{A}_{-}^{(2)} \mathbf{u}_{h}^{1, m}\right] d s
$$

is replaced by

$$
\int_{\Gamma_{0, m}^{(2)}} \phi_{\ell}(\mathbf{x})\left[\mathbf{A}_{n}^{(2)} \mathbf{u}_{R}^{0, m}\right] d s
$$

where, again, $\mathbf{A}_{n}^{(2)}$ is the boundary normal flux Jacobian matrix given in (12) while $\mathbf{u}_{R}^{0, m}$ is the out-going part of the solution along the boundary which is constructed as follows. Suppose the presumed out-going wave angle is $\theta$, defined in the same fashion as $\phi_{i}$ in Figure 7 . Then the flux in the direction of $\theta$ is $(\cos \theta, \sin \theta) \cdot \mathbf{F}$ with its 
Jacobian matrix being $\mathbf{A}_{\theta}=\cos \theta \mathbf{A}_{\mathbf{1}}+\sin \theta \mathbf{A}_{\mathbf{2}}$. Let $\mathbf{E}_{\theta}$ be a matrix whose columns are eigenvectors of $\mathbf{A}_{\theta}$, i.e.,

$$
\mathbf{A}_{\theta}=\mathbf{E}_{\theta} \mathbf{\Lambda} \mathbf{E}_{\theta}^{-1},
$$

where $\Lambda$ is the diagonal eigenvalue matrix. Then

$$
\mathbf{u}_{R}^{0, m}=\mathbf{E}_{\theta} \mathbf{I}^{+} \mathbf{E}_{\theta}^{-1} \mathbf{u}_{h}^{0, m}
$$

is the solution to be used in the edge integral. Here $\mathrm{I}^{+}$is a diagonal matrix whose diagonal values are 1 and 0 according to the corresponding eigenvalues in $\Lambda$ being positive and negative respectively. This is equivalent to decomposing the numerical solution $\mathbf{u}_{h}^{0, m}$ into eigenvector components of $\mathbf{A}_{\theta}$ and keeping only the outgoing components in $\mathbf{u}_{R}^{0, m}$. In terms of the expansion coefficient vector $\hat{\mathbf{C}}^{n m}$, the finite wave model leads to the following equation to determine the reflection coefficients:

$$
\left(\mathbf{B}^{(2)} \otimes \mathbf{A}_{+}^{(2)}\right) \hat{\mathbf{C}}^{0, m}+\left(\mathbf{B}^{\prime(2)} \otimes \mathbf{A}_{-}^{(2)}\right) \hat{\mathbf{C}}^{1, m}=\left(\mathbf{B}^{(2)} \otimes \mathbf{A}_{n}^{(2)}\right) \hat{\mathbf{C}}_{R}^{0, m}
$$

where

$$
\hat{\mathbf{C}}_{R}^{0, m}=\left(\mathbf{I} \otimes \mathbf{E}_{\theta} \mathbf{I}^{+} \mathbf{E}_{\theta}^{-1}\right) \hat{\mathbf{C}}^{0, m} .
$$

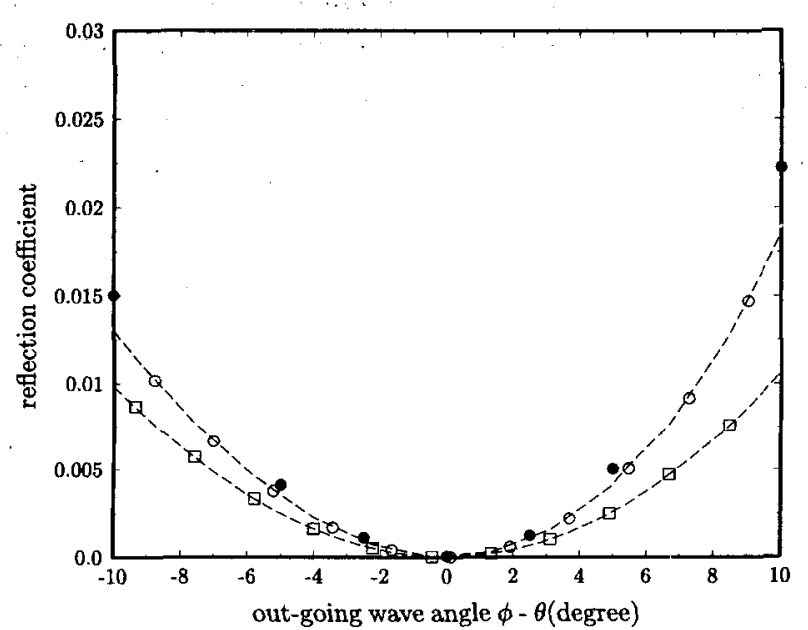

Figure 10. Reflection coefficient of the finite wave model boundary condition. Circle: $M=0$; square: $M=0.5$. $\omega h=0.5$. Open symbols are the results of discrete analysis and closed symbols are results from direct numerical simulation. Dashed line is the prediction according to the solution of partial differential equations.

In figure 10 , we show the reflection coefficient of the finite wave model boundary condition with respect to an out-going acoustic wave. The non-dimensional frequency is $w h=0.5$. The presumed out-going wave angle was taken to be $\theta=\pi / 4$ while the actual out-going wave angle $\phi_{i}$ was varied. As we can see, the reflections are effectively annihilated for actual out-going waves with an angle $\phi_{i}$ close to $\theta$.

\section{Conclusions}

We have presented a discrete analysis of three nonreflecting boundary conditions for the discontinuous Galerkin method. For the Perfectly Matched Layer absorbing boundary condition, we computed the discrete wavenumbers and eigenfunctions of the semi-discrete equation. Our analysis showed that the reflections at the interface of Euler and PML domains caused by the discretization process were reduced and approached to zero as the resolution of the scheme was increased. For the characteristic and finite wave model non-reflecting boundary conditions, the discrete reflection errors converged to those predicted by the non-discrete partial differential equations and agreed well with results of discrete numerical simulations.

ACKNOWLEDGEMENT F. Q. Hu was supported by NASA Grant NAG-1-01044.

\section{References}

[1] H. Atkins, Continued development of the discontinuous Galerkin method for computational aeroacoustics applications, AIAA-paper 97-1581, 1997.

[2] H. Atkins and J. Casper, Nonreflective boundary conditions for high-order methods, AIAA Journal, Vol. 32, 512-518, 1994.

[3] Atkins and C.-W. Shu, Quadrature-free implementation of discontinuous Galerkin methods for hyperbolic equations, $A I A A$ Journal, Vol. 36, 775-782, 1998.

[4] R. L. Higdon, "Numerical absorbing boundary conditions for the wave equation", Math Computation, Vol. 49, 65-90, 1987.

[5] F. Q. Hu, A Stable, Perfectly Matched Layer for linearized Euler Equations in unsplit physical variables, Journal of Computational Physics, Vol. 173, 455-480, 2001.

[6] F. Q. Hu and H. L. Atkins, Eigensolution analysis of the discontinuous Galerkin method with non-uniform grids, part I: one space dimension. Journal of Computational Physics, Vol. 182, 516-545, 2002.

[7] F. Q. Hu and H. L. Atkins, Two-dimensional wave analysis of the discontinuous Galerkin method with non-uniform grids and boundary conditions, AIAA-paper 2002-2514, 2002.

[8] K.W. Thompson, "Time-dependent boundary conditions for hyperbolic systems II", Journal of Computational Physics, Vol. $89,439-461,1990$.

[9] M. B. Giles, "Non-reflecting boundary conditions for Euler equation calculations", AIAA Journal, Vol. 28, 2050-2058, 1990.

\section{Appendix}




\section{A1. Definition of matrices in (24)}

The mass and "stiffness" matrices for equation (24) are defined as follows:

$$
\begin{aligned}
\mathbf{Q} & =\left\{q_{\ell \ell^{\prime}}\right\}_{L \times L}, q_{\ell^{\prime} \ell}=\int_{\Omega} \phi_{\ell} \phi_{\ell^{\prime}} d \mathbf{x} \\
\mathbf{Q}_{x}=\left\{q_{\ell \ell^{\prime}}\right\}_{L \times L}, q_{\ell^{\prime} \ell} & =\int_{\Omega} \frac{d \phi_{\ell}}{d x} \phi_{\ell^{\prime}} d \mathbf{x} \\
\mathbf{Q}_{y}=\left\{q_{\ell \ell^{\prime}}\right\}_{L \times L}, q_{\ell^{\prime} \ell} & =\int_{\Omega} \frac{d \phi_{\ell}}{d y} \phi_{\ell^{\prime}} d \mathbf{x} \\
\mathbf{B}^{(i)}=\left\{b_{\ell \ell^{\prime}}\right\}_{L \times L}, b_{\ell^{\prime} \ell} & =\int_{\Gamma^{(i)}} \phi_{\ell} \phi_{\ell^{\prime}} d s \\
\mathbf{B}^{\prime(i)}=\left\{b_{\ell^{\prime}}^{\prime}\right\}_{L \times L}, b_{\ell \ell^{\prime}}^{\prime} & =\int_{\Gamma^{(i)}} \phi_{\ell} \phi_{\bar{\ell}^{\prime}}^{-} d s
\end{aligned}
$$

where $\phi_{\ell^{\prime}}$ in matrix $\mathbf{B}^{\prime}$ denotes the basis function of the exterior element along edge $\Gamma^{(i)}$.

A2. Non-discrete analysis of the characteristic and the finite wave model non-reflecting boundary conditions

(1) Characteristic non-reflecting boundary boundary condition

In the non-discrete limit, the characteristic boundary condition can be shown to be equivalent to applying $\mathbf{A}_{n}^{-} \mathbf{u}=\mathbf{0}$ at the non-reflecting boundary. Using eigen-solutions of the Euler equation (1), the reflection coefficient of an out-going acoustic wave is found to be

$$
\beta_{r}=\frac{1-\cos \phi_{i}}{1-\cos \phi_{r}}
$$

and that of an out-going vorticity wave is

$$
\beta_{r}=\frac{\sin \phi_{i}}{1-\cos \phi_{r}}
$$

where $\phi_{i}$ and $\phi_{r}$ are defined in Figure 7. It is to be noted that the incident and reflected angles are related as

$$
\frac{\sin \phi_{r}}{1+M \cos \phi_{r}}=\frac{\sin \phi_{i}}{1+M \cos \phi_{i}}
$$

for the out-going acoustic waves and

$$
\frac{\sin \phi_{r}}{1+M \cos \phi_{r}}=\frac{\tan \phi_{i}}{M}
$$

for the out-going vorticity waves.
(2) Finite wave model

Let $\theta$, defined in the same fashion as $\phi_{i}$ in Figure 7, be the presumed angle of the out-going wave used in the construction of the solution at the boundary. Then the reflection coefficient of an out-going acoustic wave is

$$
\beta_{r}=\frac{1-\cos \left(\phi_{i}-\theta\right)}{1-\cos \left(\phi_{r}-\theta\right)}
$$

and the reflection coefficient of an out-going vorticity wave is

$$
\beta_{r}=\frac{\sin \left(\phi_{i}-\theta\right)}{1-\cos \left(\phi_{r}-\theta\right)}
$$

